PROCEEDINGS OF THE

AMERICAN MATHEMATICAL SOCIETY

Volume 127, Number 4, April 1999, Pages 1209-1213

S 0002-9939(99)05216-8

\title{
THE MOD 2 HOMOLOGY OF BSO
}

\author{
CLAUDINA IZEPE RODRIGUES
}

(Communicated by Ralph Cohen)

ABstract. This note is about a set of generators to the mod 2 homology of BSO.

\section{INTRODUCTION}

It is well known that $H_{*}\left(B O ; \mathbb{Z}_{2}\right)$ is a polynomial ring, $\mathbb{Z}_{2}\left[x_{i} \mid i \geq 1\right]$, where $x_{i} \in H_{i}\left(B O ; \mathbb{Z}_{2}\right)$. The generators $x_{i}$ may be chosen to come from the nonzero classes in $H_{i}\left(B O_{1} ; \mathbb{Z}_{2}\right)$ under the stabilization map, and in particular, $x_{i}=f_{*}\left[R P^{i}\right]$, where $f: R P^{i} \rightarrow B O$ classifies the usual line bundle over projective space.

The corresponding dual basis of $H^{*}\left(B O ; \mathbb{Z}_{2}\right)$ is usually denoted by $s_{w}$, where $w=$ $\left(i_{1}, \ldots, i_{r}\right)$. If the splitting principle is used to write universal Stiefel-Whitney classes $w_{i}$ formally as the $i$-th elementary symmetric function in 1-dimensional classes $t_{1}, t_{2}, \ldots$, then $s_{w}=\sum t_{1}^{i_{1}} t_{2}^{i_{2}} \ldots t_{r}^{i_{r}}$ is the smallest symmetric function containing the given monomial. In particular, if $y=\sum a_{j_{1} \ldots j_{s}} x_{1}^{j_{1}} \ldots x_{s}^{j_{s}} \in H_{*}\left(B O ; \mathbb{Z}_{2}\right)$, the coefficients are $a_{j_{1} \ldots j_{s}}=s_{\left(j_{1}, \ldots, j_{s}\right)}[y]$.

S. Papastavridis [1] has shown that $H_{*}\left(B S O ; \mathbb{Z}_{2}\right)$ is also a polynomial ring, $\mathbb{Z}_{2}\left[y_{i} \mid i>1\right]$, which is described as a subring of $H_{*}\left(B O ; \mathbb{Z}_{2}\right)$ by choosing classes $y_{i}$ as polynomials in the $x_{j}$. (Note: It is well known that $H^{*}\left(B S O ; \mathbb{Z}_{2}\right)$ is the quotient of $H^{*}\left(B O ; \mathbb{Z}_{2}\right)$ by the ideal generated by $w_{1}$. Dually, $H_{*}\left(B S O ; \mathbb{Z}_{2}\right)$ can be identified with a subring of $H_{*}\left(B O ; \mathbb{Z}_{2}\right)$.) Papastavridis' choices of the classes $y_{i}$ are clearly algebraically independent and hence give a subring of $H_{*}\left(B O ; \mathbb{Z}_{2}\right)$ which has precisely the same dimension as $H_{*}\left(B S O ; \mathbb{Z}_{2}\right)$. The hard part of his argument is to see that the classes $y_{i}$ lie in $H_{*}\left(B S O ; \mathbb{Z}_{2}\right)$.

The purpose of this paper is to simplify Papastavridis' argument. For any integer $n>1$, one chooses a pair of integers $(j, k)$ with $j+k=n$ by

$$
\left\{\begin{array}{l}
(j, k)=(0, n) \quad \text { if } \quad n=2^{r}, \\
(j, k)=\left(2^{r}, 2^{r+1} s\right) \quad \text { if } \quad n=2^{r}(2 s+1), s>0 .
\end{array}\right.
$$

Then, let $z_{n} \in H_{n}\left(B O ; \mathbb{Z}_{2}\right)$ be the classes $f_{*}\left[R P^{j} \times R P^{k}\right]$ where $f: R P^{j} \times R P^{k} \rightarrow$ $B O$ classifies the bundle $\xi_{1} \oplus \xi_{2} \oplus\left(\xi_{1} \otimes \xi_{2}\right)$ with $\xi_{i}$ being the usual line bundle over the $i$-th factor. Because the given bundle is orientable, it is clear that $z_{n} \in$ image $\left(H_{n}\left(B S O ; \mathbb{Z}_{2}\right) \rightarrow H_{n}\left(B O ; \mathbb{Z}_{2}\right)\right)$, and our main result is

Theorem. $H_{*}\left(B S O ; \mathbb{Z}_{2}\right)=\mathbb{Z}_{2}\left[z_{n} \mid n>1\right]$.

Received by the editors June 17, 1997.

1991 Mathematics Subject Classification. Primary 55R40.

(C)1999 American Mathematical Society 
Additionally, one has:

Fact. The classes $z_{n}=f_{*}\left[R P^{j} \times R P^{k}\right]$ coincide with Papastavridis' classes $y_{n}$.

I wish to express my thanks to Professor R. E. Stong for suggesting this problem.

\section{Proof of the Theorem}

It is clear that one has a homomorphism

$$
\varphi: \mathbb{Z}_{2}\left[u_{n} \mid n>1\right] \rightarrow H_{*}\left(B S O ; \mathbb{Z}_{2}\right) \subset H_{*}\left(B O ; \mathbb{Z}_{2}\right)
$$

defined by $\varphi\left(u_{n}\right)=z_{n}=f_{*}\left[R P^{j} \times R P^{k}\right]$, and in every dimension, $H_{*}\left(B S O ; \mathbb{Z}_{2}\right)$ and the polynomial ring have the same dimension as the $\mathbb{Z}_{2}$ vector space. To prove the theorem, it suffices to see that the classes $z_{n}$ are algebraically independent. This is immediate from:

\section{Lemma.}

$$
z_{n}=\left\{\begin{array}{lcc}
x_{n}+\text { decomposables } & \text { if } & n=2^{r}(2 s+1), \\
x_{n / 2}^{2} & \text { if } \quad n=2^{r} .
\end{array}\right.
$$

Proof. Let $H^{*}\left(R P^{j} \times R P^{k} ; \mathbb{Z}_{2}\right)=\mathbb{Z}_{2}[\alpha, \beta] /\left(\alpha^{j+1}=0, \beta^{k+1}=0\right)$, where $\operatorname{dim} \alpha=$ $\operatorname{dim} \beta=1$. The Stiefel-Whitney class of $\xi_{1} \oplus \xi_{2} \oplus\left(\xi_{1} \otimes \xi_{2}\right)$ is $(1+\alpha)(1+\beta)(1+\alpha+\beta)$. Then for $n=2^{r}(2 s+1)$,

$$
\begin{aligned}
s_{n} & =\alpha^{n}+\beta^{n}+(\alpha+\beta)^{n} \\
& =\left(\begin{array}{c}
2^{r}(2 s+1) \\
2^{r}
\end{array}\right) \alpha^{2^{r}} \beta^{2^{r+1} s},
\end{aligned}
$$

which is nonzero. For $n=2^{r}, \alpha=0$, and $H^{*}\left(R P^{n} ; \mathbb{Z}_{2}\right)=\mathbb{Z}_{2}[\beta] /\left(\beta^{n+1}=0\right)$, with the Stiefel-Whitney class of the bundle being $(1+\beta)^{2}$. Then

$$
s_{w}\left((1+\beta)^{2}\right)= \begin{cases}0 & \text { if } \quad w \neq\left(w^{\prime}, w^{\prime}\right), \\ s_{w^{\prime}}\left((1+\beta)^{2}\right) & \text { if } \quad w=\left(w^{\prime}, w^{\prime}\right),\end{cases}
$$

giving $z_{n}=x_{n / 2}^{2}$.

\section{PApastavridis' Classes}

To verify that $z_{n}=y_{n}$, as defined by Papastavridis, requires a lot of unpleasant calculation. Not only is one showing that $y_{n}$ belongs to $H_{n}\left(B S O ; \mathbb{Z}_{2}\right)$, but one is identifying the given class. Since this is obvious for $n=2^{r}$, one need only consider $n=2^{r}(2 s+1)$. The goal is to verify that $s_{(a, b, c)}\left[z_{n}\right]$, with $0 \leq a \leq b \leq c$, is given by Papastavridis' formula,

$$
\left\{\begin{array}{l}
\left(\begin{array}{c}
b-1 \\
2^{r}-a-1
\end{array}\right) \quad \text { if } \quad 2^{r} \leq b \text { and } 0 \leq a<2^{r}, \\
\left(\begin{array}{c}
b-1 \\
r\left(2^{r}-a\right)
\end{array}\right) \quad \text { if } \quad 0<b<2^{r}, 0 \leq a<2^{r} \text { and } a+r\left(2^{r}-a\right) \leq b, \\
0 \\
\text { otherwise. }
\end{array}\right.
$$

It is, of course, clear that $s_{w}\left[z_{n}\right]=0$ if $w=\left(i_{1}, \ldots, i_{r}\right)$ with $r>3$, since the defining bundle has dimension 3 . 
Here we are going to verify that $s_{(a, b, c)}\left[z_{n}\right]$ is given by the above formula only in case $0<a<b<2^{r}, c<2^{r+1} s$. In this case we have

$$
\begin{aligned}
s_{(a, b, c)}\left[z_{n}\right] & =\left\{\alpha^{a} \beta^{b}(\alpha+\beta)^{c}+\alpha^{a} \beta^{c}(\alpha+\beta)^{b}+\alpha^{b} \beta^{c}(\alpha+\beta)^{a}\right. \\
& \left.+\alpha^{b} \beta^{a}(\alpha+\beta)^{c}\right\}\left[R P^{2^{r}} \times R P^{2^{r+1}} s\right], \text { since } \alpha^{c}=0, \\
& =\left[\left(\begin{array}{c}
c \\
2^{r}-a
\end{array}\right)+\left(\begin{array}{c}
b \\
2^{r}-a
\end{array}\right)+\left(\begin{array}{c}
a \\
2^{r}-b
\end{array}\right)+\left(\begin{array}{c}
c \\
2^{r}-b
\end{array}\right)\right] \bmod 2 .
\end{aligned}
$$

Next, observe that

$$
\left(\begin{array}{c}
a \\
2^{r}-b
\end{array}\right)
$$

is the coefficient of $x^{2^{r}-b}$ in the binomial expansion of $(1+x)^{a}$, and $(1+x)^{a}=$ $\frac{(1+x)^{2^{r+1} s}}{(1+x)^{b+c-2^{r}}}$, with $b+c-2^{r}-1>0$. Recall that $\frac{1}{(1+y)^{t+1}}=\sum_{j=0}^{\infty}\left(\begin{array}{c}t+j \\ j\end{array}\right) y^{j}$.

Then

$$
\left(\begin{array}{c}
a \\
2^{r}-b
\end{array}\right) \equiv\left(\begin{array}{c}
b+c-2^{r}-1+2^{r}-b \\
2^{r}-b
\end{array}\right) \equiv\left(\begin{array}{c}
c-1 \\
2^{r}-b
\end{array}\right) \quad \bmod 2 .
$$

So, it is clear that

$$
\begin{aligned}
\left(\begin{array}{c}
c \\
2^{r}-b
\end{array}\right)+\left(\begin{array}{c}
a \\
2^{r}-b
\end{array}\right) & \equiv\left(\begin{array}{c}
c \\
2^{r}-b
\end{array}\right)+\left(\begin{array}{c}
c-1 \\
2^{r}-b
\end{array}\right) \\
& \equiv\left(\begin{array}{c}
c-1 \\
2^{r}-b-1
\end{array}\right)=\left(\begin{array}{c}
2^{r+1} s+2^{r}-a-b-1 \\
2^{r+1} s-a
\end{array}\right)
\end{aligned}
$$

and this is the coefficient of $x^{2^{r+1} s-a}$ in the binomial expansion of $(1+x)^{c-1}$, where

$$
\begin{aligned}
(1+x)^{c-1} & =\frac{(1+x)^{2^{r+1}} s(1+x)^{2^{r}}}{(1+x)^{a+b+1}} \\
= & \left\{\sum_{m=0}^{s}\left(\begin{array}{c}
s \\
m
\end{array}\right) x^{2^{r+1} s-2^{r+1} m}+\sum_{m=0}^{s}\left(\begin{array}{c}
s \\
m
\end{array}\right) x^{2^{r+1} s-2^{r+1} m+2^{r}}\right\} \\
(\mathrm{A}) & \times\left\{\sum_{l=0}^{\infty}\left(\begin{array}{c}
a+b+l \\
l
\end{array}\right) x^{l}\right\} .
\end{aligned}
$$

Now, if we take the product of any term in (A) by the complementary term in (C), the coefficient is

$$
\left(\begin{array}{l}
s \\
m
\end{array}\right)\left(\begin{array}{c}
2^{r+1} m+b \\
2^{r+1} m-a
\end{array}\right) \equiv 0 \bmod 2,
$$

since $b<2^{r}$. (Obs: if $m=0$ in $(\mathrm{A}), 2^{r+1} s>2^{r+1}-a$.) Next, observe that all the powers in the expansion (B) for $m>0$ are between $2^{r+1}(s-1)+2^{r}$ and $2^{r+1} s$, moreover, we have $2^{r+1}(s-1)+2^{r}<2^{r+1} s-a<2^{r+1} s$, since $2^{r}>a>0$. For 
$m=0$, the power is $2^{r+1} s+2^{r}$, so it is bigger than $2^{r+1} s$. Therefore,

$$
\begin{gathered}
\left(\begin{array}{c}
c \\
2^{r}-b
\end{array}\right)+\left(\begin{array}{c}
a \\
2^{r}-b
\end{array}\right)=\left\{\sum_{m=1}^{s}\left(\begin{array}{c}
s \\
m
\end{array}\right)\left(\begin{array}{c}
2^{r+1} m-2^{r}+b \\
2^{r+1} m-2^{r}-a
\end{array}\right)\right\} \\
=\left\{\sum_{m=1}^{s}\left(\begin{array}{c}
s \\
m
\end{array}\right)\right\}\left(\begin{array}{c}
b \\
2^{r}-a
\end{array}\right) \equiv\left(\begin{array}{c}
b \\
2^{r}-a
\end{array}\right) \bmod 2 .
\end{gathered}
$$

Thus, we can see immediately that

$$
s_{(a, b, c)}\left[z_{n}\right]=\left(\begin{array}{c}
c \\
2^{r}-a
\end{array}\right) \quad \bmod 2 .
$$

Now, writing $2^{r}-a=2^{j}+t, 0 \leq t<2^{j}$, with $r\left(2^{r}-a\right)=t$ as in Papastavridis [1], we have $c=2^{r+1} s+2^{j}+t-b$. So, we can look at $\left(\begin{array}{c}c \\ 2^{r}-a\end{array}\right)$ as the coefficient of $x^{2^{r}-a}$ in the binomial expansion of

$$
(1+x)^{c}=\frac{(1+x)^{2^{r+1}} s(1+x)^{2^{j}}}{(1+x)^{b-t}}
$$

Hence, it follows that

$$
s_{(a, b, c)}\left[z_{n}\right]=\left\{\left(\begin{array}{c}
2^{j}+b-1 \\
2^{j}+t
\end{array}\right)+\left(\begin{array}{c}
b-1 \\
t
\end{array}\right)\right\} \bmod 2 .
$$

Next, we can write $b=a+b^{\prime}$ with $0<b^{\prime}<2^{r}-a=2^{j}+t$, since $a<b<2^{r}$. Thus, we get

$$
\begin{aligned}
\left(\begin{array}{c}
2^{j}+b-1 \\
2^{j}+t
\end{array}\right) & =\left(\begin{array}{c}
2^{j}+2^{j}\left(2^{r-j}-1\right)-t+b^{\prime}-1 \\
2^{j}+t
\end{array}\right) \\
& \equiv\left\{\begin{array}{l}
\left(\begin{array}{c}
b-1 \\
t
\end{array}\right) \bmod 2 \text { if } 0<b^{\prime} \leq t, \\
0 \bmod 2 \quad \text { if } t<b^{\prime}<2^{j}+t
\end{array}\right.
\end{aligned}
$$

and

$$
\left(\begin{array}{c}
b-1 \\
t
\end{array}\right)=\left(\begin{array}{c}
2^{j}\left(2^{r-j}-1\right)-t+b^{\prime}-1 \\
t
\end{array}\right) \equiv 0 \quad \bmod 2 \text { if } t<b^{\prime}<2 t .
$$

Finally, since

$$
0<b^{\prime}<2 t \Longleftrightarrow a+2 r\left(2^{r}-a\right)>b
$$

and

$$
2 t \leq b^{\prime}<2^{j}+t \Longleftrightarrow a+2 r\left(2^{r}-a\right) \leq b,
$$

we conclude that

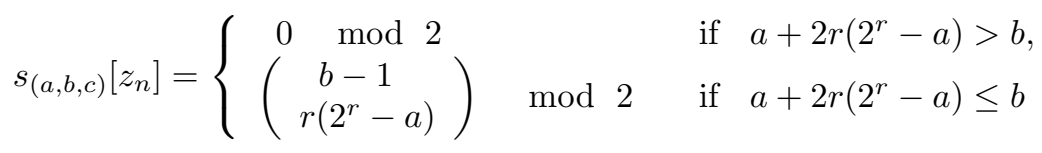

as in [1].

Since we have checked one of the more difficult cases, one now can write down the other possibilities for $(a, b, c)$ using similar calculations. 


\section{REFERENCES}

[1] S. Papastavridis, The image of $H_{*}\left(B S O ; \mathbb{Z}_{2}\right)$ in $H_{*}\left(B O ; \mathbb{Z}_{2}\right)$, Proc. Amer. Math. Soc., Vol. 107, No. 4, 1989, pp. 1071-1073. MR 90m:55017

imeCC - Departamento de Matemática, Universidade Estadual de Campinas, Caixa Postal - 6065, 13081-970 - CAMPINAS - S.P., Brasil

E-mail address: claudina@ime.unicamp.br 